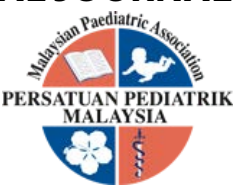

\title{
RELEVANCE OF SCREENING FOR URINARY TRACT INFECTION IN WELL INFANTS WITH PROLONGED JAUNDICE: A PROSPECTIVE COHORT STUDY
}

\author{
Caroline Siew Yin Eng, Thalitha Vijayakulasingam, Boon Hong Ng, Maryam Jamilah Surdi \\ Roslan, Muhammad Zamakhshari Zainal Abidin, Ming Lee Lee
}

\begin{abstract}
Background: Recent retrospective studies suggest irrelevance of urine screening for neonate with prolonged jaundice. We re-evaluated the incidence of urinary tract infection (UTI) among these infants, their renal outcome and evaluated the cost incurred. Methods: This is a prospective cohort study. Asymptomatic, prolonged jaundiced infants with unconjugated hyperbilirubinemia were screened for evidence of UTI as per local guidelines. Infants with pyuria would have urine sent for culture and sensitivity. Unit cost was referenced from hospital purchase. Statistical analysis was performed using SPSS 24.0. Results: A total of 291 cases were analyzed. Majority were term infants (93.8\%). The commonest cause of prolonged jaundice was breast milk jaundice, hence an incidence rate of $0.34 \%$. Only one infant persistently showed single uropathogen on urinary culture with concurrent pyuria. Urinary structures were normal on ultrasonography and there was no evidence of renal cortical scarring. No recurrence of UTI documented in the first year of life. Each "clean-catch" urinalysis costed RM7. This unit cost escalated to RM37 for catheterized sample. A negative urine culture costed RM28 while a positive culture twice this price. The average cost effectiveness ratio (ACER) in this study was RM5856.56 per detection of case. Conclusion: Incidence of UTI is low. In our study, an undesirable outcome is negligible. Unnecessary parental anxiety from the potentially laborious procedure could be avoided. This study refutes previous literature to include such screening in prolonged jaundice as this may well be irrelevant.
\end{abstract}

Keywords:

Prolonged Jaundice, Unconjugated

hyperbilirubinemia, Urinary Tract Infection

\section{Introduction}

Urinary tract infection (UTI) has been cited as a cause of prolonged jaundice in infants. The association between UTI and prolonged jaundice remains controversial. International and local clinical guidelines continue to recommend screening for urine infection in all infants presenting with prolonged jaundice $[1,2]$.

Appropriate method of collection is important to ensure accurate interpretation of result. However, urinary screening at local health clinics by acceptable methods could be laborious. In reality, many practitioners resorted to the easiest method of urinary collection by the urine bag which conferred many false positive results.
Received: 16 September 2019; Accepted revised

manuscript: 21 November 2019

Published online: 15 December 2019
Inadvertently, the patients and their parents will be subjected to repeat sampling procedures which can contribute to immense distress to both parties; physically and emotionally.

In the past decades, high UTI rates had been cited in literature [3-6]. Renal scarring as a potential implication of UTI in the newborn justified the need for screening in this group for many decades. However, more recent studies have illustrated conflicting evidence on the association between prolonged jaundice and urinary tract

Department of Paediatrics, Hospital Tuanku Ja'afar, Jalan Rasah 70300 Seremban, Malaysia

Corresponding author: Dr. Caroline Siew Yin Eng, Department of Paediatrics, Hospital Tuanku Ja'afar, Jalan Rasah 70300 Seremban, Malaysia Tel: +6067684391 ext. 5158/5157; Email: carolinesyeng@gmail.com 
infection. In two separate retrospective studies, English researchers quoted low UTI rate and advocated for the exclusion of UTI screening that has been imposed on otherwise well infants with prolonged jaundice $[7,8]$. Findings from the latter studies are promising but it is not uncommon that retrospective studies could be limited by a lack of standardization and missing data. Furthermore, these data may not reflect population from different demographic and socioeconomic background.

This is important especially when this new understanding could imply a change in practice. The present study evaluate the incidence of UTI among infants with prolonged jaundice in a developing country, underlying congenital abnormalities of the kidney and urinary tract (CAKUT) and subsequent renal scarring among newborns who screened positive. Reproducing similar results in a developing country would add weight to the notion against inclusion of screening. In addition, costs of the present screening procedures were also evaluated.

\section{Materials and Methods}

\section{Study design and study participants}

The study was a prospective, cross sectional study prevalent in otherwise well newborns with prolonged jaundice, predominantly unconjugated hyperbilirubinaemia. Prolonged jaundice is defined as visible jaundice (or serum bilirubin $>85 \mathrm{mmol} / \mathrm{L}$ ) that persists beyond 14 days of life in a term infant or 21 days in a preterm infant [9]. We recruited all infants who fulfilled the inclusion criteria from the Special Care Nursery at Hospital Tuanku Ja'afar Seremban as well as infants presented to all Community Health Centres within the district of Seremban. Seremban is one of the 7 districts in the state of Negeri Sembilan, covering approximately $14 \%$ of the area in the state and is also where the capital of the state is located. Recruitment took place from January 2018 till December 2018 and the infants recruited were followed up till 1 year of age as per protocol.

Exclusion criteria set for the study were infants with fever, irritability, history of poor feeding or any other clinical features of possible sepsis at presentation or became unwell within 72 hours of presentation, infants with poor weight gain (defined as failure to achieve expected weight gain in the second week of life by average 10$20 \mathrm{~g} / \mathrm{kg} /$ day or $1-3 \%$ of body weight/day) [9], infants with concomitant dysmorphism, infants who were admitted for other medical indications and went on to develop jaundice that persisted beyond days 14 or 21, whichever applicable and infants with prolonged jaundice secondary to a surgical cause such as biliary atresia (conjugated hyperbilirubinaemia).

Infants recruited in the study were subjected to a screening procedure in tandem with the recommendation from the national paediatric working group [2]. This ensured that the study methodology did not differ much from practice in real life. Both blood and urine samples were collected for serum bilirubin, full blood count with reticulocyte count, peripheral blood film for morphology, glucose-6-phosphate dehydrogenase (G6PD) qualitative testing, thyroid function and urinalysis. Infants who were unwell or those with predominantly conjugated hyperbilirubinaemia were excluded and referred for appropriate management.

In this study, caregivers were allowed to collect urine by applying a sterile self-adhesive urine bag apart from "clean-catch" or "in-out catheterization method. Bag sampling of urine for urinalysis is practical in a busy clinic setup but its utility is limited only to identification of normal urine sample. Urine samples were analysed using dipstick and read by analyzer. Negative urinalyses from a bag sample were regarded as not suggestive for UTI. Infants with sample tested positive were referred within the same week to the prolonged jaundice clinic at the hospital, simply because the workload and time factor at the health centres made it logistically impractical for the test to be repeated at the local level. At the hospital, urine was re-sampled for urinalysis and culture either via "clean-catch" or "in-out catheterization method. Infants who fulfilled definition of UTI were treated accordingly with antibiotics and their serum bilirubin level monitored accordingly. UTI is defined as the presence of at least 105 colony forming units (CFU) per milliliter $(\mathrm{ml})$ of a single uropathogen and concomitant pyuria which refers to presence of 5 or more leucocyte per high power field [1012]. All infants treated for UTI were subjected to renal ultrasonography to look for presence of congenital abnormalities of kidney and urinary tract (CAKUT) and scheduled for dimercaptosuccinic acid (DMSA) scan at not less than 6 months of life. They were also followed up for recurrence of UTI up to 1 year of age. Infants diagnosed with dilated urinary tracts on ultrasonography were treated accordingly based on severity of their urinary tract dilatation 
(postnatal Urinary Tract Dilatation (UTD) Classification) [13]. The study was approved by the national research ethics committee. Consent was obtained from parents/ legal carers.

\section{Statistical methodology}

Based on recent reported UTI rate of $0.21 \%$ in literature, sample size estimation was calculated using the population proportion formulae [14]. If the Type 1 error probability and precision are both 0.05 , we would need to study 255 infants. With an additional of $10 \%$ dropout rate, the estimated sample size was 281 infants.

We used standard descriptive statistics to characterize our study participants. All continuous parametric variables were expressed as mean \pm standard deviation while those of nonparametric in nature were expressed as median along with their respective first and third quartiles, whenever appropriate. Incidence of UTI amongst neonate with prolonged jaundice was described in percentages (\%). Unit cost of urinary testing depending on method of collection for both urinalysis as well as urine culture and sensitivity were referenced from hospital purchasing records. This is reflective of cost incurred in a public funded health institution. Average cost-effectiveness ratio (ACER) was evaluated by dividing the total implementing cost with the number of cases fulfilled definition of UTI. All data submitted were analysed using SPSS version 24.0.

\section{Results}

A total of 291 infants were recruited from our institution and 11 health centres within the same district. Background demographic data are shown in Table 1.

Table 1. Demographic characteristics of study population

\begin{tabular}{ll}
\hline & Number (\%) \\
\hline $\begin{array}{l}\text { Gender } \\
\text { Female }\end{array}$ & $152(52.2)$ \\
\hline Ethnicity & $139(47.8)$ \\
Malay & $249(85.6)$ \\
Chinese & $14(4.8)$ \\
Indian & $22(7.6)$ \\
Others & $6(2.0)$ \\
\hline Weight at birth (kg) & \\
Below 2.0 & $4(1.4)$ \\
$2.0-3.0$ & $150(51.5)$ \\
$3.0-4.0$ & $133(45.7)$ \\
Above 4.0 & $4(1.4)$ \\
\hline Gestation at birth (weeks) & $18(6.2)$ \\
Less than 37 & $233(80.0)$ \\
37 - 40 & $40(13.8)$ \\
More than 40 & \\
\hline Feeding methods & $210(72.2)$ \\
Exclusively breastfeeding & $81(27.8)$ \\
Mixed feeding (formula and & \\
breastmilk) &
\end{tabular}

All of them were otherwise well and thriving. Mean total serum bilirubin was $175.2 \pm 55.1$ $\mathrm{mmol} / \mathrm{L}$. The commonest cause of prolonged unconjugated hyperbilirubinaemia was breastmilk jaundice (62.2\%).

Three quarter of the cohort (216 infants; 74.2\%) had their urine dipstick screened negative at their first encounter for prolonged jaundice work-up.
The remaining $25.8 \%$ or 75 infants were referred to the prolonged jaundice clinic for a repeat urine testing which included urine culture and sensitivity; of which 14 consented for an "in-out" catheterization and the rest via "clean-catch" method. Fifty-nine out of these 75 infants were discharged as re-testing demonstrated neither pyuria nor yield positive growth. Sixteen infants had positive growth with or without pyuria. 
Nevertheless, all these infants (16 infants) remained asymptomatic. They underwent a third re-testing as soon as result of their second testing was known. At their third screening, 15 infants had urine collected via "in-out" catheterization and 1 via "clean-catch" method. Only three samples showed positive growth with single uropathogen but only 1 with concomitant pyuria (Refer Table 2).

Table 2. Culture results demographic characteristics of study population

\begin{tabular}{llll}
\hline No. & $\begin{array}{l}\text { Patient's age at } \\
\text { presentation }\end{array}$ & $\begin{array}{l}\text { Urine culture result } \\
\left(\mathbf{3}^{\text {rd }} \text { testing) }\right.\end{array}$ & Urinalysis \\
\hline 1. & Day 15 of life & Escherichia coli & Absent pyuria \\
2. & Day 22 of life & Escherichia coli & Absent pyuria \\
3. & Day 16 of life & Escherichia coli & Leucocyte $1+$ \\
\hline
\end{tabular}

Although all 3 infants were treated for presumed UTI following the third testing, only 1 patient (Patient 3) demonstrated significant single uropathogen and pyuria. This translates to an UTI incident rate of $0.34 \%$ amongst this group of well infants presenting with prolonged unconjugated hyperbilirubinaemia based on the definition of UTI.

One infant had transient hydronephrosis that completely resolved upon a repeat ultrasonography after 3 months. DMSA scans at no earlier than 6 months of life did not demonstrate evidence of renal scarring in any of these infants. All of them remained well with no recurrence of UTI throughout their first year of life.

Unit cost incurred differed for various methods of urinalysis. Unit cost for a positive urine culture and sensitivity doubled that of a negative culture due to testing against various antibiotics panel. Unit cost for the various urinary testing is tabulated in Table 3.

Table 3. Unit cost

\begin{tabular}{ll}
\hline Nature of urinary testing & Unit cost (MYR) \\
\hline Urinalysis from a bag collected sample & 7.30 \\
Urinalysis from a "clean catch" catheterized sample & 7.05 \\
Urinalysis from an "in-out" catheterized sample & 37.18 \\
$\begin{array}{l}\text { Urine culture and sensitivity: } \\
\text { - Negative culture }\end{array}$ & 17.20 \\
- Positive culture (average; range depending on type of & $45.98 ; 35.61-55.38$ \\
$\quad$ organism cultured) & \\
\hline
\end{tabular}

MYR: Malaysian Ringgit

In this study, a total of 382 urinalyses and 91 urine cultures and sensitivity were performed. Utilizing the unit cost tabulated in Table 3, estimated cost of screening is as shown in Table 4 .

Table 4. Projected cost of screening based on calculated unit cost in our cohort

\begin{tabular}{lll}
\hline Nature of urinary testing & $\begin{array}{l}\text { No. of test } \\
\text { performed }\end{array}$ & $\begin{array}{l}\text { Screening cost } \\
\text { (MYR) }\end{array}$ \\
\hline Urinalysis from a bag collected sample & 234 & 1708.20 \\
Urinalysis from a "clean catch" catheterized sample & 116 & 817.80 \\
Urinalysis from an "in-out" catheterized sample & 32 & 1189.76 \\
$\begin{array}{l}\text { Urine culture and sensitivity: } \\
\bullet \quad \text { Negative culture }\end{array}$ & 71 & 1221.20 \\
$\bullet \quad$ Positive culture (average; range depending on & 20 & 919.60 \\
$\quad$ type of organism cultured) & 473 & 5856.56 \\
\hline
\end{tabular}

MYR: Malaysian Ringgit 
In this study, total cost of screening for 291 infants was estimated to be RM5856.56. Hence, average cost-effectiveness ratio (ACER) in this study was RM5856.56 as only 1 case fulfilled definition of UTI.

\section{DISCUSSION}

The association between hyperbilirubinaemia and UTI in neonates has been described many decades ago. As early as 1928, Professor Görter and his colleague published their series of 3 cases of infants less than a year with jaundice and pyelitis [15]. Subsequently, several other series similarly had illustrated such association $[16,17]$. The "hepatotoxin" from infection led to hyperbilirubinaemia from various mechanism, biliary stasis and disruption of the enzymatic pathway for bilirubin conjugation are some of the mechanisms proposed. In all these series, the children were described to be unwell with pyrexia.

Until today, National Institute for Health and Clinical Excellence (NICE) recommends urinary testing as part of the evaluation for infants with prolonged jaundice. This recommendation is an initiative to identify infants at risk of UTI so that treatment could be administered early. With recent studies showing the contrasting low detection rate of UTI in well infants with prolonged jaundice, questions have been raised on the relevance of screening practices. Unnecessary parental anxiety from the potentially laborious procedure could be avoided, especially when this could well affect the mental health of the new mother who is already overwhelmed with her new role. Our study concurred with recent literature. We demonstrated reproducibility of low incidence rate of UTI $(0.34 \%)$ in a planned prospective study. About three quarter of our study population was exclusively breastfed. Prevalence of breast milk jaundice was high in our cohort and such affected infants are usually very well except for the tinge of jaundice that may persist well into the second or third months of life. This minute fraction could possibly reflect asymptomatic bacteriuria in otherwise very well infants. Asymptomatic bacteriuria is not an uncommon phenomenon in early infancy; incidence reported to be low at $1.3 \%$ from a study involving 151 healthy preterm babies [18]. This condition does not warrant treatment unless they are subjected to urological procedures or in children undergoing kidney transplantation $[19,20,21]$.

Economic burden has been a real concern to most health institution. Due to the short term nature of this condition and the impracticality involved in gauging health value especially byproxy, the utility of incremental cost-effectiveness ratio (ICER) in this situation is limited. Average cost effectiveness ratio (ACER) instead is a direct way of illustrating the cost involved per detection of case. ACER in our study was RM5856.56 per case detected. Taking into consideration of modern literature that increasingly supports against treatment of asymptomatic bacteriuria and the low detection rate, cost of screening demonstrated in our study is high. Omitting this as a routine practice may not result in an untoward outcome. Advanced perinatal care over the years has improved the detection of infants with congenital abnormality of kidney and urinary tract (CAKUT). It is also important to acknowledge that febrile UTI warrants treatment instead and treating asymptomatic bacteriuria may expose patients to unnecessary antibiotics and unwarranted follow-up renal imaging.

\section{Conclusion}

Findings from this suggest that screening for UTI amongst well infants with prolonged jaundice is no longer relevant. Such low detection rate and lack of negative outcome of not screening do not justify such high screening cost. We proposed that urinary screening for UTI is reserved for symptomatic unwell infants.

\section{Competing interest}

None declared

\section{Acknowledgements}

We would like to extend our gratitude to Madam $\mathrm{Ku}$ Nurhasni $\mathrm{Ku}$ Abd Rahim from the Health Technology Assessment Unit, Medical Development Division, Ministry of Health of Malaysia for her invaluable advice and comments on this study. We gratefully acknowledge the permission granted by State Health Director for his permission to involve the health clinics in Negeri Sembilan. Finally, our appreciation to the Director General of Health Malaysia for his permission to publish this article. 


\section{References}

[1] Rodie ME, Barclay A, Harry C, Simpson J. NICE recommendations for the formal assessment of babies with prolonged jaundice: too much for well infants? Arch Dis Child. 2011;96:112-3.

[2] Prolonged Jaundice in newborn infants. In: Muhammad Ismail $\mathrm{HI}$, Mohd Ibrahim $\mathrm{H}, \mathrm{Ng}$ HP, Thomas T, editors. Paediatric Protocols For Malaysian Hospitals $4^{\text {th }}$ Edition. Kuala Lumpur: Malaysian Paediatric Association 2018. p. 146-53.

[3] Garcia FJ, Nager AL. Jaundice as an early diagnostic sign of urinary tract infection in infancy. Pediatrics. 2002;109:846-51.

[4] Ghaemi S, Fesharaki RJ, Kelishadi R. Late onset jaundice and urinary tract infection in neonates. Indian J Pediatr. 2007;74:139-41.

[5] Najati N, Gharebaghi MM, Mortazavi F. Underlying etiologies of prolonged icterus in neonates. Pakistan Journal of Biological Sciences. 2011;13:711-4.

[6] Pashapour N, Nikibahksh AA, Golmohammadlou S. Urinary tract infection in term neonates with prolonged jaundice. Urol J. 2007;4(2):91-4.

[7] Steadman S, Ahmed I, McGarry K, Rasiah SV. Is screening for urine infection in well infants with prolonged jaundiced required? Local review and meta-analyses of existing data. Arch Dis Child. 2016;101:614-9.

[8] Chowdhury T, Kisat H, Tullus K. Does UTI cause prolonged jaundice in otherwise well infants? Eur J Pediatr. 2015;174(7):971-3.

[9] Gomella TL, Eyal FG, Bany-Mohammed F. Gomella's Neonatology Management, Procedures, On-call Problems, Diseases and Drugs. $8^{\text {th }}$ Edition 2020.

[10] Roberts KB, Downs SM, Finnell SM, Hellerstein S, Shortliffe LD, Wald ER et al. Urinary tract infection: clinical practice guideline for the diagnosis and management of the initial UTI in febrile infants and children 2 to 24 months. Pediatrics. 2011;128(3):595-610.
[11] Jones KV, Banerjee J, Boddy SA, Grier D, Jadresic L, Larcombe J, et al. NICE guideline on urinary tract infection in children: Diagnosis, treatment and long term management. 2007.

[12] Kass EH. Bacteriuria and the diagnosis of infections of the urinary tract: With observations on the use of methionine as a urinary antiseptic. AMA Arch Intern Med. 1957;100(5):709-14.

[13] Nguyen HT, Benson CB, Bromley b et al. Multidisciplinary consensus on the classification of prenatal and postnatal urinary tract dilation (UTD classification system). J Pediatr Urol. 2014;10(6):982-98.

[14] Lemeshow S, Hosmer DW, Klar J, Lwanga SK. Adequacy of sample size in health studies. World Health Organization. John Wiley \& Sons 1990.

[15] Gorter, E., and Lignac, G. 0. E. On pyelitis complicated by jaundice. Arch Dis Child. 1928;3:232.

[16] Ng SH, Rawstron JR. Urinary tract infections presenting with jaundice. Arch Dis Child. 1971;46:173-6.

[17] Arthur, A. B., and Wilson, B. D. R. Urinary infection presenting with jaundice. British Medical Journal. 1967;1:539.

[18] Moncrieff M, Bamford M, Benson J, Bodden J. Asymptomatic bacteriuria in healthy preterm babies. Arch Dis Child. 1980;55(9):723-5.

[19] Kotagiri P, Chembolli D, Ryan J, Hughes PD, Toussaint ND. Urinary tract infections in the first year post-kidney transplantation: potential benefits of treating asymptomatic bacteriuria. Transplant Proc. 2017;49(9):20705.

[20] Sharifian M, Rees L, Trompeter RS. High incidence of bacteriuria following renal transplantation in children. Nephrol Dial Transplant. 1998;13(2):432-5.

[21] Bonket G, Pickard R, Bartoletti R, Bruyère $F$, Geerlings SE, Wagenlehner $F$, et al. EAU guidelines on urological infections. Arnhem, Neth: European Association of Urology. 2017. 